\title{
Intermittent Audio Failure Analysis of a Remote Speaker-Microphone for a Two-Way Radio
}

\author{
K. H. Leong - R. H. A. Latiff - F. Yusof - C. C. Ooi • \\ M. R. A. Rahman
}

Submitted: 2 December 2015/Published online: 29 December 2015

(C) ASM International 2015

\begin{abstract}
This paper presents a case study of an intermittent audio failure analysis of a remote speakermicrophone module for a two-way radio. A root cause analysis was undertaken to identify probable causes of the intermittent failure, followed by a series of experiments to determine the strength and the intermittent audio failure load of cable components and the fully assembled cable. The combined experimental and finite element results demonstrated that the main contributor of the intermittent audio failure was the micro surface cracks on the copper conductor strands. In addition, the combination of the component materials and design of the cable have also contributed to the non-uniform state of residual stress induced in the copper conductors which have reduced the ability of the copper conductors to withstand the normal handling load under the influence of micro surface cracks.
\end{abstract}

Keywords Electronic cable intermittent contact . Experimental analysis - Finite element analysis

K. H. Leong · R. H. A. Latiff $(\bowtie) \cdot$ F. Yusof .

M. R. A. Rahman

School of Mechanical Engineering, University of Science

Malaysia, Nibong Tebal, 14300 Penang, Malaysia

e-mail: rizmanhariz@gmail.com

F. Yusof

e-mail: mefeizal@usm.my

C. C. Ooi

Motorola Solutions Malaysia Sdn. Bhd., Plot 2, Bayan Lepas Technoplex Industrial Park, Mukim 12 SWD, 11900 Penang, Malaysia

\section{Introduction}

The reliability of land mobile electronic audio device, such as a two-way radio, is extremely important in public health and security operations, particularly for law enforcers, search and rescue personnel, and armed forces operations [1]. The two-way radio reliability and efficient operation is equally important to consumer and industrial business operations where ground radio communications are usually conducted using portable two-way radios. As the communications operations involve critical situations relating to health and safety of personnel and the success of business operations, it is paramount that the two-way radio can meet a basic function of transmitting and receiving audio signals within a zero tolerance to failure requirement. To meet the strict requirement of the application of the two-way radio, the environmental reliability design conditions are usually based on requirements set by [2]. Although a two-way radio may meet the stipulated design requirements, intermittent or breakdown of audio communications can still occur due to degradation of materials, such as worn connection joints, broken wires, and corroded connections [3]. Loading anomalies subjected to the two-way radio can also contribute to intermittent failure, as discussed in $[4,5]$.

A two-way radio typically operates through a half-duplex mode in which a user can talk to the radio which then transmits the signal to another radio on the same frequency. The main components of a typical two-way radio include an electronic radio-frequency and controller systems connected to a microphone and a speaker $[6,7]$. A user can directly talk to the microphone and audible messages can be received through the speakers. However, for rugged applications, a remote speaker-microphone (RSM) can be connected to the two-way radio through a universal connector. A typical RSM connected to a two-way radio is 

connected to a two-way radio (right); (b) a user wearing an RSM and a two-way radio [8]
Fig. 1 (a) An RSM (left)

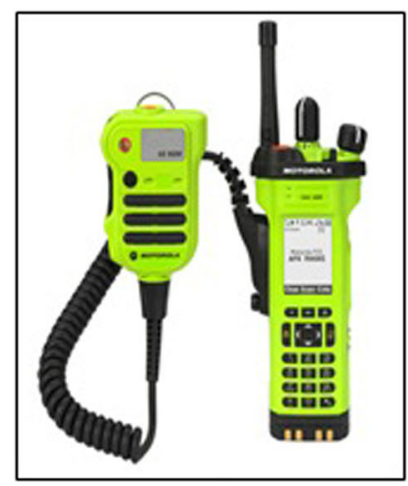

(a)

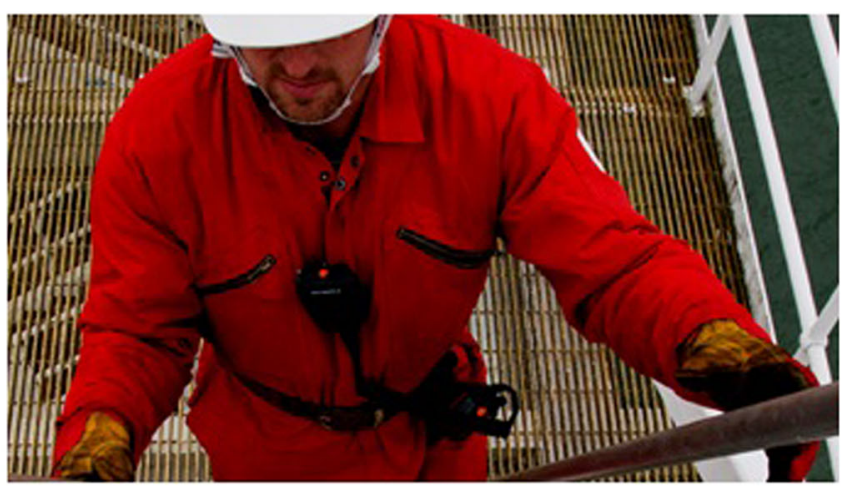

(b) shown in Fig. 1a, while Fig. 1b shows a user using an RSM connected to a two-way radio.

This paper is centered on the intermittent failure of an RSM connected to a two-way radio. The RSM module is a type of multi-core cable designed for audio applications. The cable is usually reinforced with Kevlar for heavy-duty application and is also a standard in military-grade multicore cables [9]. Usually, these cables are put through rigorous testing before they are deemed fit for use [10].

An intermittent audio failure of an RSM module manufactured by a third-party supplier for a commercially available two-way radio has been tested in this failure analysis. The failure report as received by [11] indicated that a number of remote speaker-microphone units from the same supplied batch had developed intermittent audio during usual application. The report indicated that intermittent audio failures were detected by the users and there were no circumstances of mishandling the RSM cable by the users.

From the RSM module design specification [12], the reliability requirements stated that the RSM cable should be able to withstand a cyclic bending load of $5 \mathrm{~N}$ at 20 cycles/min without impairing the audio function of the RSM module. However, a more important criterion would be the maximum static deformation load permissible for the RSM cable, which was not stated. The requirement for maximum static load was important because the intermittent failure report had indicated that failure occurred due to simple bending of the RSM cable. To understand the static load that can be applied by a user, a reference human grip data applied on typical grounded environments can be referred from [13]. It indicated that the typical pull strength ranges from 249 to $165 \mathrm{~N}$ and 165 to $111 \mathrm{~N}$ for males and females, respectively. This information has been used as a reference in this analysis to assess the limit of the load that can be carried by the RSM module.

Initial investigation of the failed remote speaker-microphone units [14] through a scanning electron

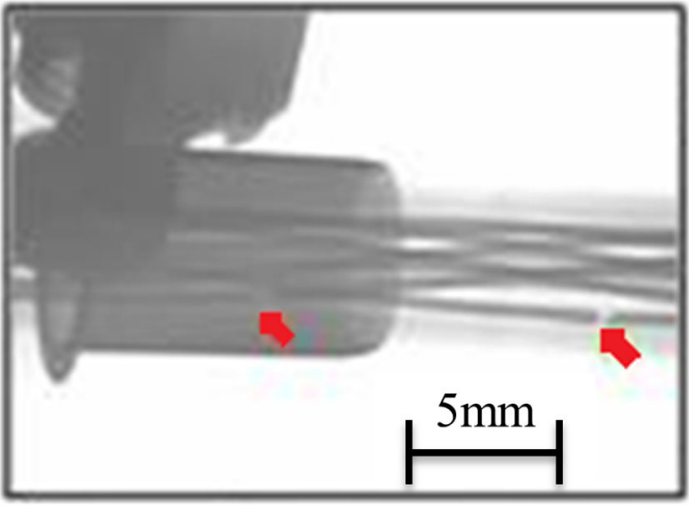

Fig. 2 Red arrows show separated conductor in the RSM cable [14] (Color figure online)

microscope showed that the copper wires within the RSM cable had separated as indicated in Fig. 2.

From this prior investigation, the intermittent audio failure analysis has been focused on ascertaining the root cause of the broken copper wires. The severity of the failure toward the safe use of the two-way radio has become a critical issue to the customer and the manufacturer as intermittent audio can endanger the life of the user or the public in cases of emergency when distress calls cannot be transmitted and received appropriately and timely. Although there was clear evidence that the conductor had separated and caused intermittent audio failure, the cause of this breakage was still elusive.

To characterize the intermittent audio failure, a systematic failure analysis approach that was modified from [4] is shown in Fig. 3. The failure analysis flowchart shows that the focus was to determine how the multi-core cables have failed through separation of the copper conductors. The study can be divided into two categories involving the manufacturing process investigation and the design investigation. The failure analysis effort was initiated by a physical inspection of the wire cable and its components to determine compliance of the 


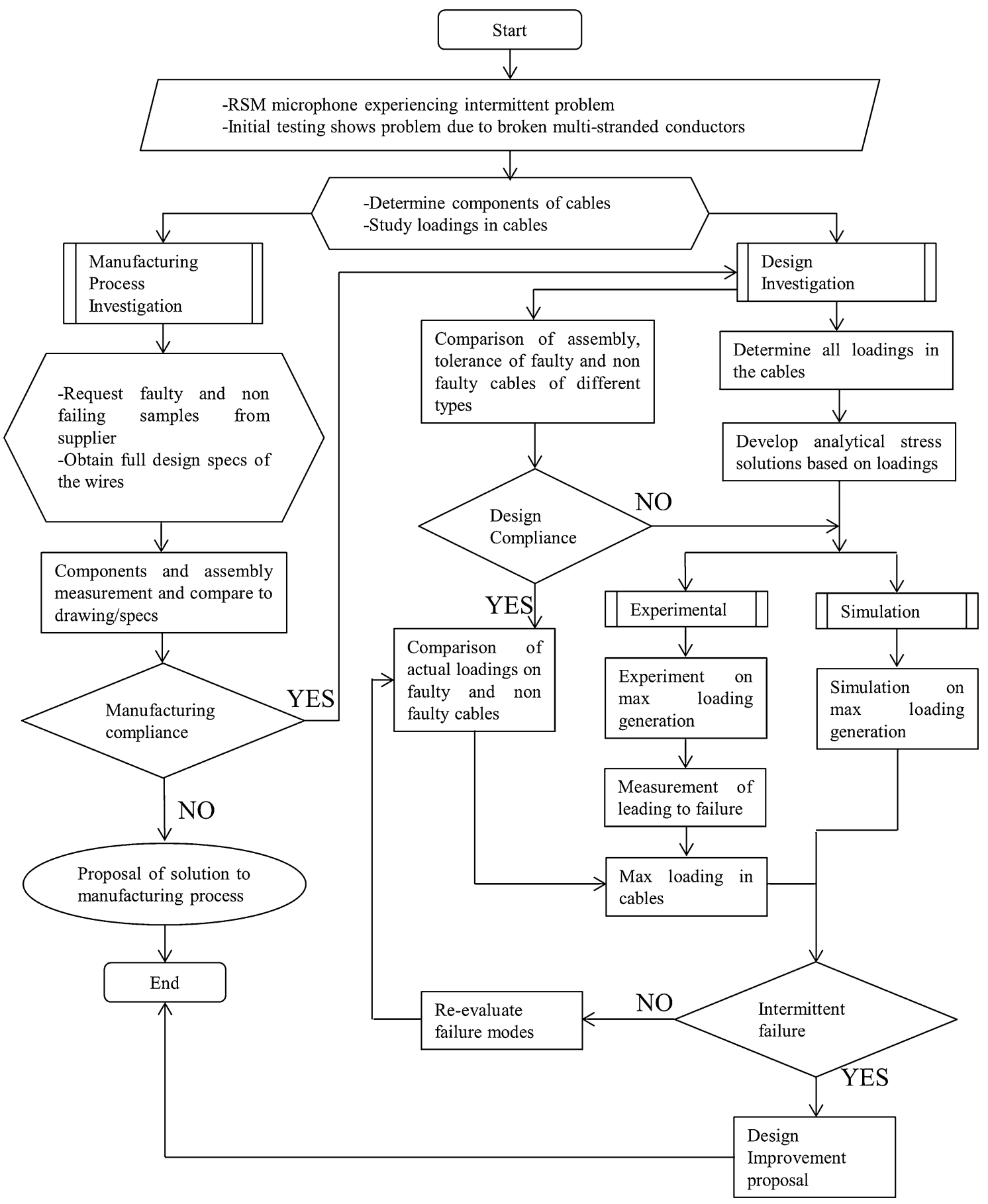

Fig. 3 Failure analysis flowchart for the remote speaker-microphone

RSM cable and the components to the design specifications [12]. Next, the strength of the RSM cable and the individual components that make up the cable were determined via a universal testing machine for tension and bending loadings. A series of finite element analyses of the RSM cable and the individual components of the RSM cable were undertaken to simulate the intermittent audio failure. Finally, the results from the experimental testing data and the finite element analysis results were both used to formulate a predictive assessment method. This method is used to predict the occurrence of intermittent audio failure. 


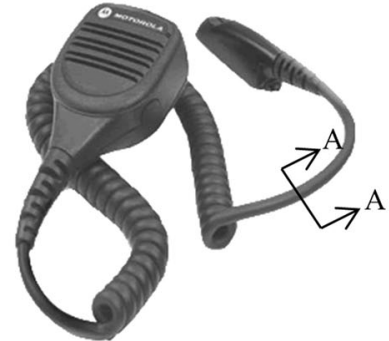

(a)

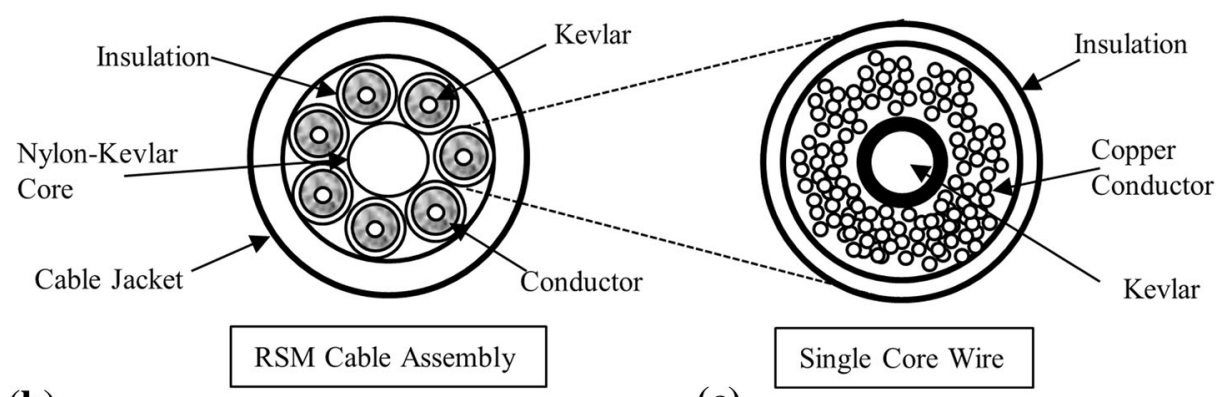

(c)

Fig. 4 (a) A sample of the RSM cable experiencing intermittent audio failure; (b) schematics of a cross section of the RSM cable assembly at AA and (c) enlarged cross section of a single-core wire

Fig. 5 (a) Epoxy-encased RSM cable and (b) non-encased RSM cable
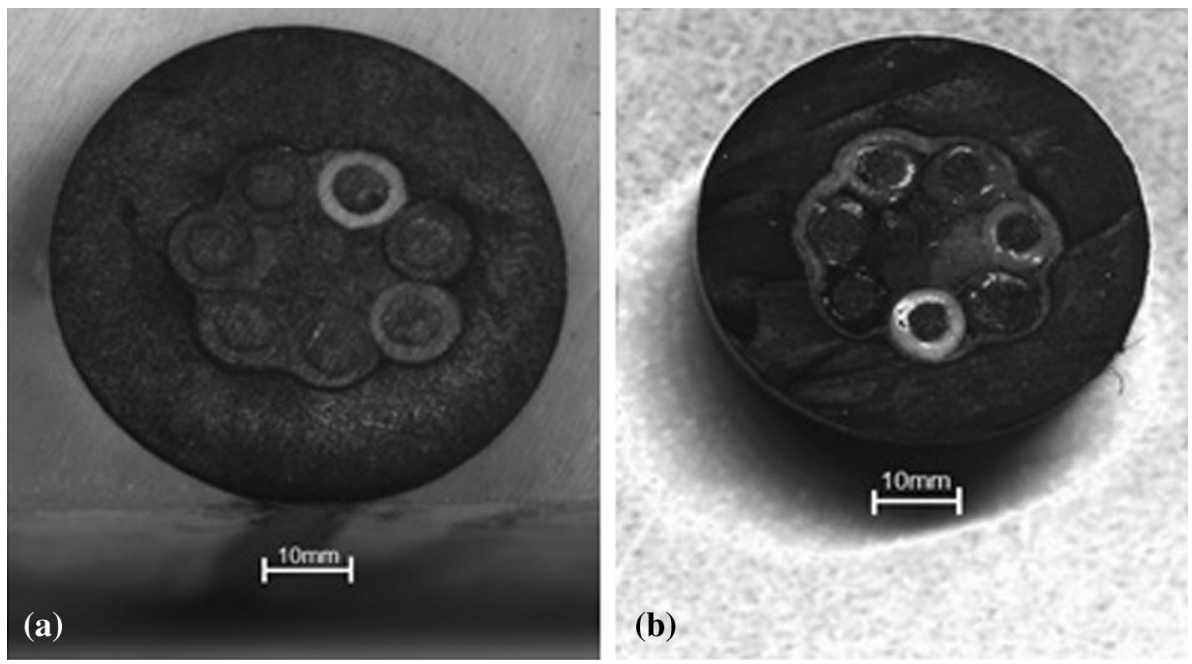

\section{Physical Examination of the RSM Cable}

The RSM is shown in Fig. 4a and the cross section A-A of the cable is depicted in Fig. 4b. The multi-core cable consisted of seven single-core wires bound together by a cable jacket with a composite Kevlar-Nylon resin at the center as shown in Fig. 4b. Each of the seven wires consisted of 49 strands of copper conductors held together by a composite Kevlar filler and insulation as shown in the enlarged cross section of a single wire in Fig. 4c.

For the physical examination of the RSM cable, a number of dissections were made on the cable. It was observed and measured that the cable components experienced some relaxation after dissection. This behavior was similar to residual stress effect in metals $[15,16]$. To quantify the cross section dimensional relaxation of RSM cable components, an epoxy-encased cable assembly specimen and non-encased cable assembly specimen were prepared, as shown in Fig. 5. The dimensions of the cable assembly components before and after relaxation were compared, as shown in Table 1.

The hardened epoxy surrounding the specimen ensures that the components of the RSM cable were not allowed to relax after dissection. The outcome of the analysis indicated that the RSM components were found to be in a compressive fit. There were no design specifications from [12] to indicate the level of compressive fit of the cable components, but it is speculated that the compressive fit was necessary for the manufacture of the RSM cable. From the four components that made up the RSM cable, the cable jacket showed the largest change in the cross section dimension before and after dissection, indicating the largest source of residual stress. It is speculated that the residual stress can be ascribed to the thermal contraction of the cable jacket.

In a different physical examination of the RSM copper strands using a stereomicroscope, scores of micro surface cracks were identified along the copper conductors. 
Table 1 Comparison of RSM cable components after cutting

\begin{tabular}{llccc}
\hline & Component list & Dimensions with relaxation $(\mathrm{mm})$ & Dimensions without relaxation $(\mathrm{mm})$ & Percentage difference $(\%)$ \\
\hline 1. & Copper conductor & 0.536 & 0.530 & +1.13 \\
2. & Insulation & 0.952 & 0.930 & +2.37 \\
3. & Kevlar & 0.154 & 0.154 & 0 \\
4. & Cable jacket & 5.661 & 5.450 & +3.87 \\
\hline
\end{tabular}
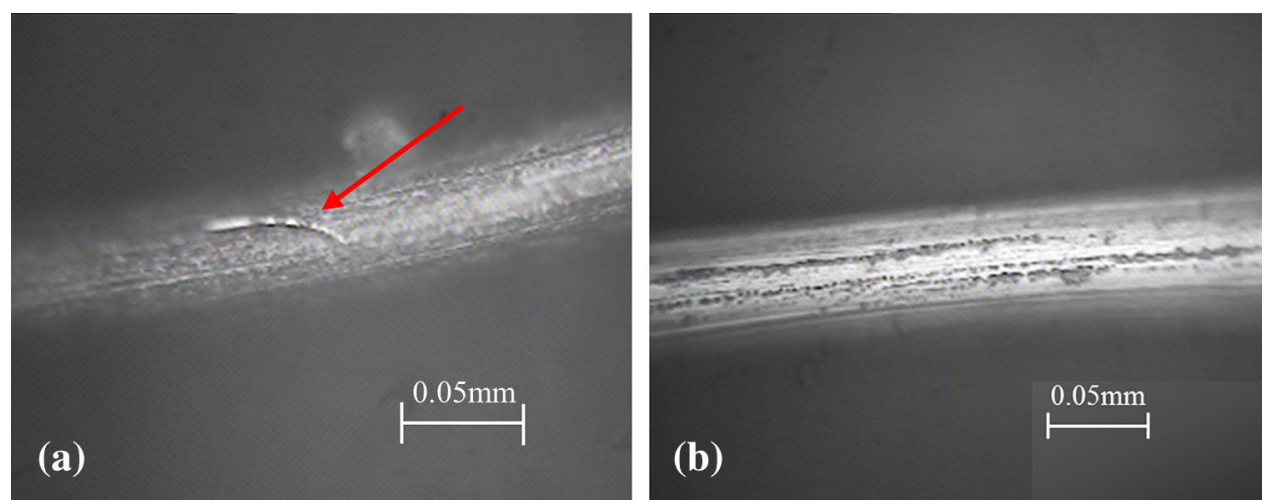

Fig. 6 Image of a copper conductor from samples of (a) intermittent failure RSM cable with surface crack of $45.6 \mu \mathrm{m}$ in length and (b) RSM cable with no intermittent failure

Figure 6a shows an example of a longitudinal surface crack on the copper conductor. The arrow indicates a scratch with a size of $45.6 \mu \mathrm{m}$. Figure $6 \mathrm{~b}$ shows a copper conductor strand from a non-faulty specimen demonstrating a crack-free surface.

From the physical examination analysis, it can be postulated that the copper strand may have separated due to residual stress, minor surface crack, or combination of both defects that led to the intermittent audio failure. Detailed experimental analysis and finite element analysis are discussed in the following sections.

\section{Experimental Analysis}

Typical loads that the RSM cable can be subjected to during normal use involve tensile, bending, and twisting loads as well as a combination of the loading modes. Based on the failure report [11], the intermittent audio failure analysis of the RSM cable was limited to direct tensile loading and tensile loading with a bending configuration.

The testing programs include testing the individual components of the RSM cable as well as the entire RSM cable assembly in order to evaluate the effect of the residual stress and micro surface cracks on the copper conductors. The experimental analysis was based on ASTM E8 standards [17] with specific reference to sections 5 and 7. For the experimental analysis, all specimens were selected from a non-defective RSM cable batch. The strain rate was set to $1 \mathrm{~mm} / \mathrm{min}$ and the data logging was set to a frequency of $5 \mathrm{~Hz}$.

To exert a maximum bending load to the RSM cable and the components, a critical bending radius was required. In general, the critical bending radius was a function of the diameter of the cable. In the current failure analysis, the bending radius of $10 \mathrm{~mm}$ has been identified experimentally to cause a maximum stress on the cable, which was also the smallest attainable bending radius obtained through bending.

Following [17], the wires were securely attached to the universal testing clamps using a snubbing device. To facilitate an in situ intermittent failure signal identification, a test board with independent light-emitting diodes (LEDs) connected to each of the individual wires in the RSM cable was supplied with constant voltage from a battery source of $3 \mathrm{~V}$ as shown in Fig. $7 \mathrm{a}$ and $\mathrm{b}$.

During testing, the test board indicated the sequence in which the copper conductors within the cable have undergone separation. As the copper conductors were separated, the circuit becomes open and the LED connected to the wire that has separated will turn off. For all the tests conducted, the intermittent contact was reproducible and was indicated by the blinking of the LEDs. All the tension and bending test results are shown together with the finite element analysis in the subsequent section. 
Fig. 7 (a) Tensile test and (b) bending test set-up
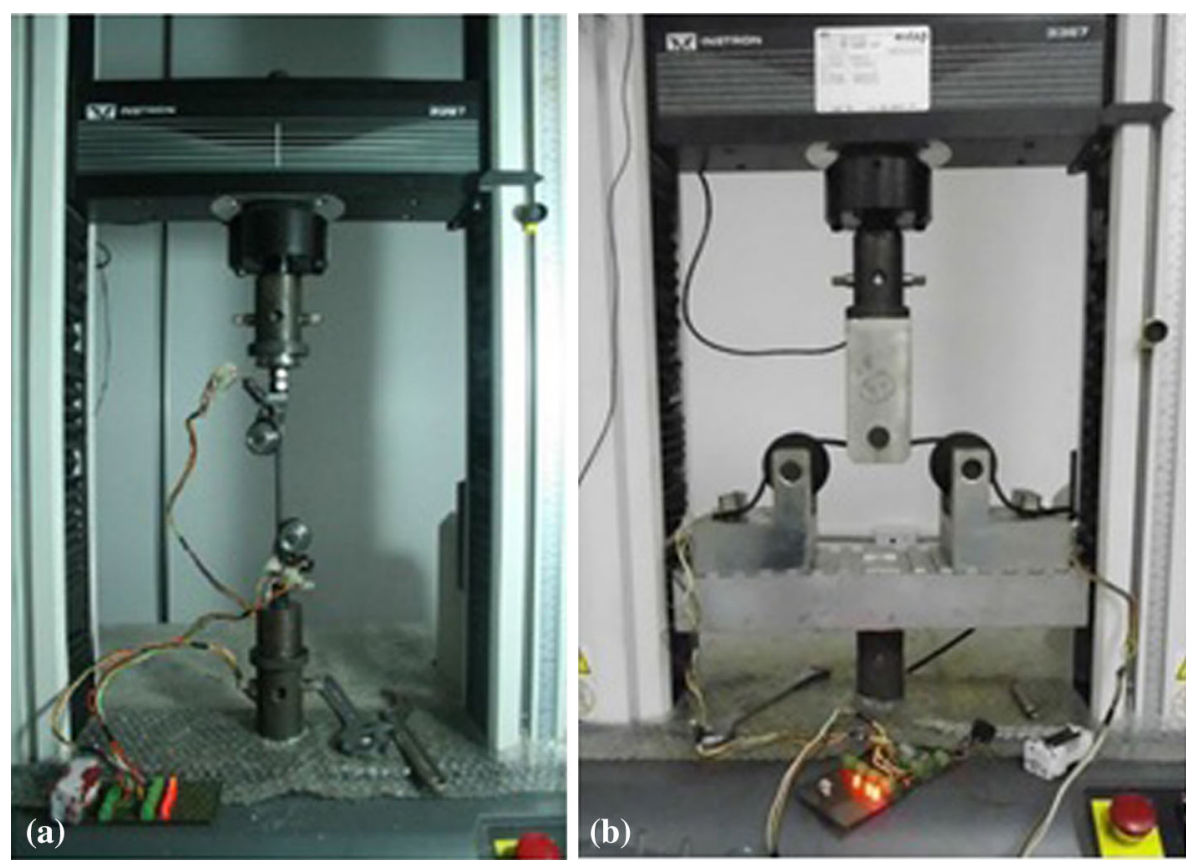

\section{Finite Element Analysis}

A commercially available multi-physics finite element code, ABAQUS [18], was used for the finite element analysis. All the components that constitute the RSM cable were modeled using reduced integration 8-node hexahedral elements as shown in Fig. 8. The origin of the model was defined at the middle of the RSM cable, $x=0, y=0$, and $z=0$ as shown in Fig. 8. The cross section A-A showed that equal-sized elements were arranged radially as focused rings in components which possess hollow cylindrical shape except Nylon-Kevlar filler core and Kevlar strands. The filler core and Kevlar strands were meshed freely across the cross section. In the axial direction, there were 36,200 elements for the tension model, while the bending model comprised 275,000 elements. Identical planar mesh as shown at A-A was constructed along the z-axis and elements were biased from the middle to the end of the RSM cable.

Frictional behaviors between the contacting components within the RSM cable were also incorporated in the present finite element model based on a Coulomb friction model by defining the friction coefficient, $\mu$, to characterize the maximum allowable shear stress across the selected interface as a fraction of contact pressure between two contacting bodies. Basically, the contacting bodies will remain attached to each other until a critical shear stress was reached. The frictional contact will govern movement between the components when the RSM cable was loaded. The frictions coefficients retrieved from [19-22] were defined for the contacting components as shown in Fig. 9.
The copper conductor, Kevlar strand, and insulation material responses were based on a Ramberg-Osgood material response to characterize the power law behavior demonstrated in the experimental analysis:

$$
\begin{aligned}
\varepsilon_{e} & =\frac{\sigma}{E} ; \quad \sigma<\sigma_{y} \\
\varepsilon_{p} & =\left(\frac{\varepsilon_{y}}{\sigma_{y}^{n}}\right) \sigma^{n} ; \quad \sigma \geq \sigma_{y} .
\end{aligned}
$$

Here, $\varepsilon_{e}, \varepsilon_{p}, \sigma_{y}$, and $\varepsilon_{y}$ are the elastic strain, plastic strain, yield stress, and yield strain, respectively. $E$ is the elastic modulus of the material and $n$ is the strain-hardening coefficient. The stress-strain relations for the materials were generalized for multi-axial stress state using the von Mises yield criterion and an associated flow rule to describe incremental irreversible deformation.

To characterize the damage initiation of the components, fracture strain in the function of stress triaxiality was used as implemented in [18]. The stress triaxiality, $\eta$, is expressed as the ratio of mean stress, $\sigma_{m}$, to equivalent von Mises stress, $q$.

$\eta=-\left(\sigma_{m} / q\right)$

To reduce the mesh dependency after damage based on strain localization, a stress-displacement response characterized by damage parameter, $d$, and equivalent plastic displacement, $\bar{u}^{\mathrm{pl}}$, was introduced to describe the strain softening of the material. Before damage initiation, $\bar{u}^{\mathrm{pl}}=0$. Once the damage initiation criterion was fulfilled where fracture strain has been reached, the plastic 

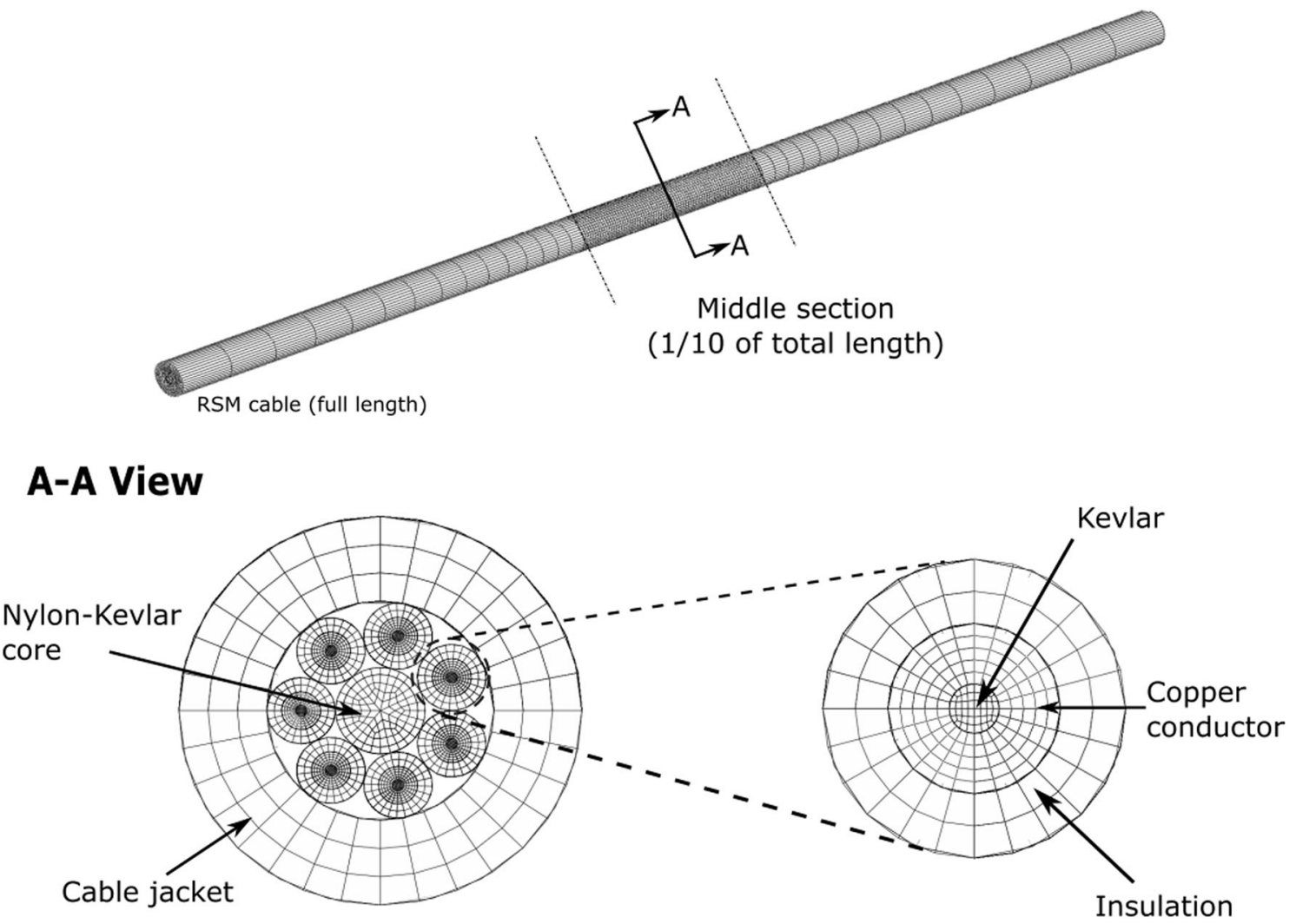

Fig. 8 Schematic of finite element mesh of full-length RSM cable (not to scale)

Fig. 9 Friction coefficients, $\mu$, defined for contacting components within the RSM cable

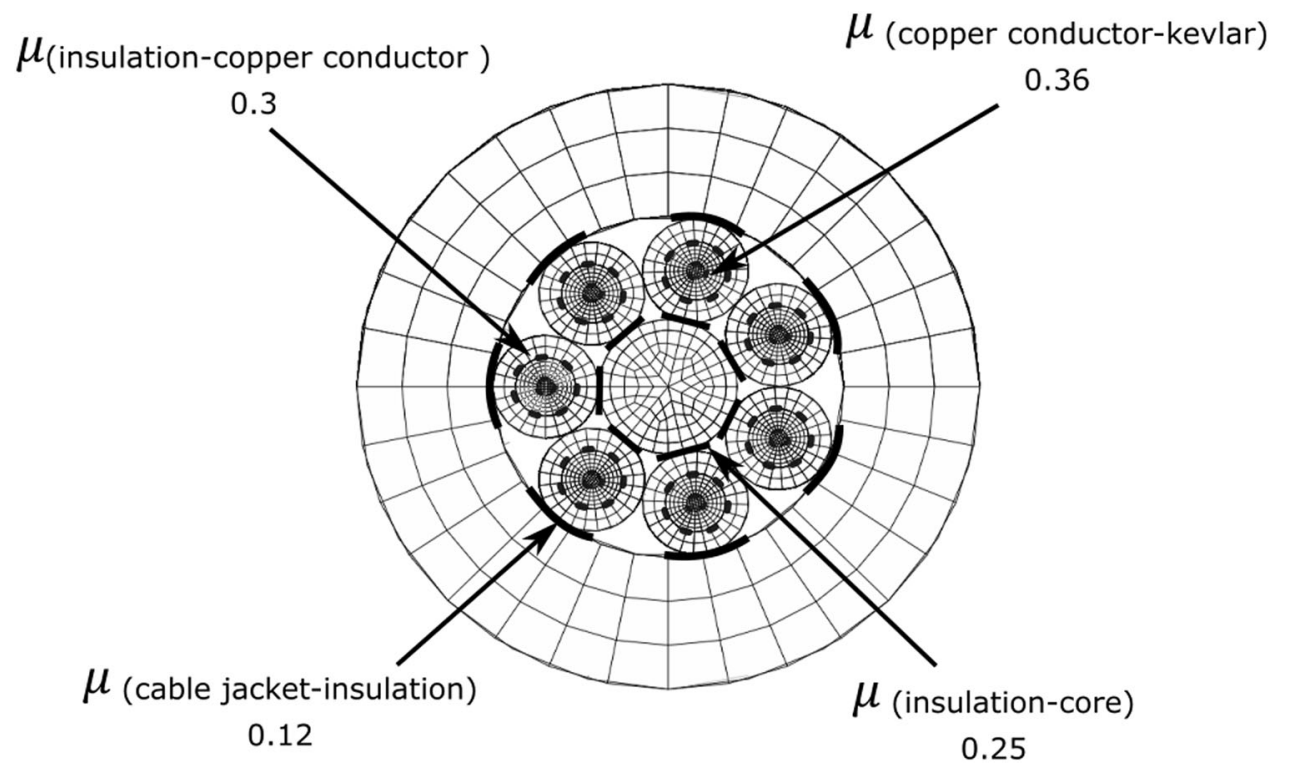

displacement, $\bar{u}^{\mathrm{pl}}$, was defined using the following evolution equation:

$\bar{u}^{\mathrm{pl}}=L \bar{\varepsilon}^{\mathrm{pl}}$,

where $L$ is the characteristic length of the element and $\bar{\varepsilon}^{\mathrm{pl}}$ is the equivalent plastic strain. On the other hand, damage parameter, $d$, can be computed through $\sigma=(1-d) \bar{\sigma}$

$(\mathrm{Eq} 4)$

Here, $\sigma$ is given as the actual true stress and $\bar{\sigma}$ is the true stress in the undamaged response. The undamaged response for the components can be predicted by extrapolating the true stress-true strain curve after necking, using a power law fit. 


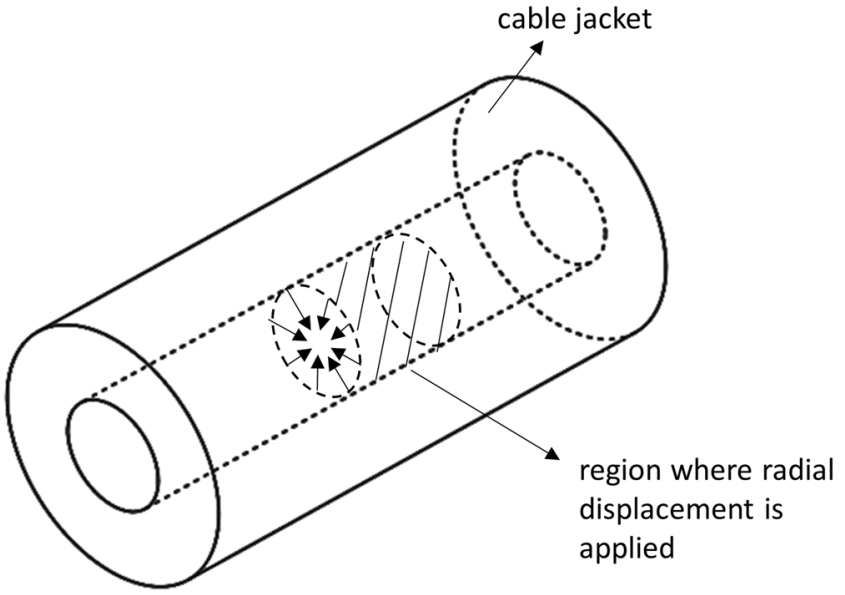

(a)

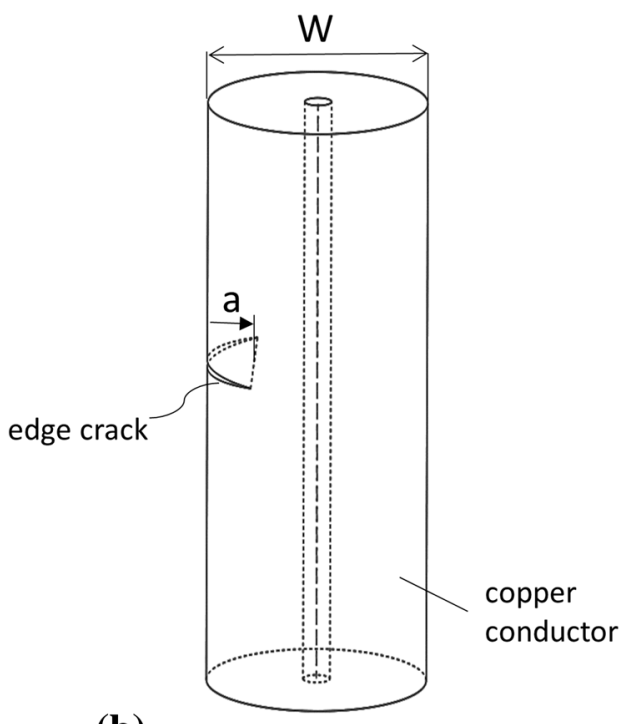

(b)

Fig. 10 (a) Radial displacement on the inner surface of cable jacket to model the compressive residual stress effect and (b) an edge crack to model micro surface crack defect

For the cable jacket and the nylon that exhibited instantaneous elastic response under large strains, a hyperelastic material behavior was used to represent the material response. The optimum hyper-elastic model for use in the analysis was based on a convergence and stability iteration which allowed the use of the Marlow strain energy potential, defined as

$U=U_{\operatorname{dev}}\left(\bar{I}_{x}\right)+U_{\mathrm{vol}}\left(J_{\mathrm{el}}\right)$,

where $U$ is the strain energy per unit of initial volume, $U_{\mathrm{dev}}$ represents the deviatoric part, and $U_{\mathrm{vol}}$ is the volumetric part. $J_{\mathrm{el}}$ is the elastic volume ratio. $\bar{I}_{x}$ is the first deviatoric strain invariant and is defined as

$\overline{I_{x}}=\overline{\lambda_{x}^{2}}+\overline{\lambda_{y}^{2}}+\overline{\lambda_{z}^{2}}$.

$\bar{\lambda}_{i}$ is the deviatoric stretch and is expressed as

$\bar{\lambda}_{i}=J^{-1 / 3} \lambda_{i}$

while $J$ is the total volume ratio and $\lambda_{i}$ represent the principal stretches. The deviatoric part of the potential was defined through the uniaxial test data from the experimental analysis, while the volumetric part $U_{\text {vol }}$ was defined through the Poisson's ratio of the material.

For the tensile loading, the boundary condition was applied as displacement, $U$, based on the experimental result onto the free end of the model, while the opposite end was constrained in the $z$-axis direction leaving the $x$ axis and the $y$-axis to move freely. The bending load boundary condition was imposed through a rigid disk modeled with a critical bending radius of $10 \mathrm{~mm}$ following the experimental set-up.
The observed modes of defect which can lead to intermittent audio failure were the residual stress and the micro surface cracks found on the copper conductors. To simulate the residual stress, the internal wall diameter of RSM cable jacket was reduced to facilitate a radial circumferential displacement around a sectional area on the wire cable mesh as shown in Fig. 10a. The circumferential compression was applied according to the limit of the design tolerance of the RSM cable jacket.

The effect of the micro surface crack observed on the copper strand can be modeled with a straight-through edge crack as shown in Fig. 10b. Several micro surface crack depths were examined according to a ratio of $a / W=0.25$ and 0.5 to represent shallow and deep micro surface crack problems.

\section{Results and Discussion}

A load-displacement curve comparison of the finite element analysis to the experimental analysis for each of the components of the RSM cable is shown in Fig. 11, which demonstrated that the difference between the experimental and finite element results is found to be within a percent of deviation. The finite element results indicated that the models developed were able to simulate the behavior of the RSM components from an elastic state to the damage process and final separation of the components.

The tensile loading analysis as shown in Fig. 11 demonstrated that damage was initiated at the Kevlar strands within the single-core wire, as shown in Fig. 4c. 


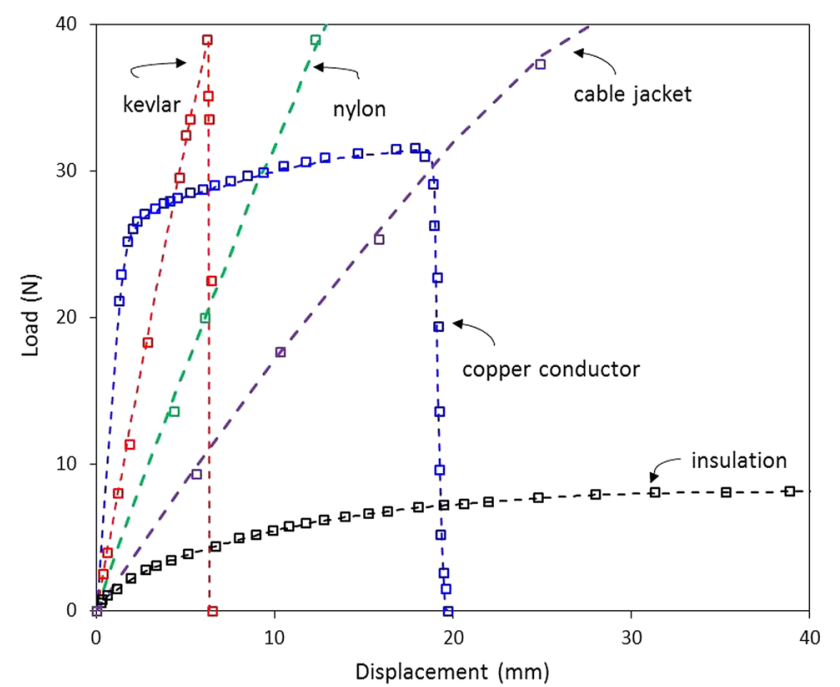

Fig. 11 Comparison of the finite element result (dashed line) to the experimental data (symbol) from tensile analysis of all the components in RSM cable

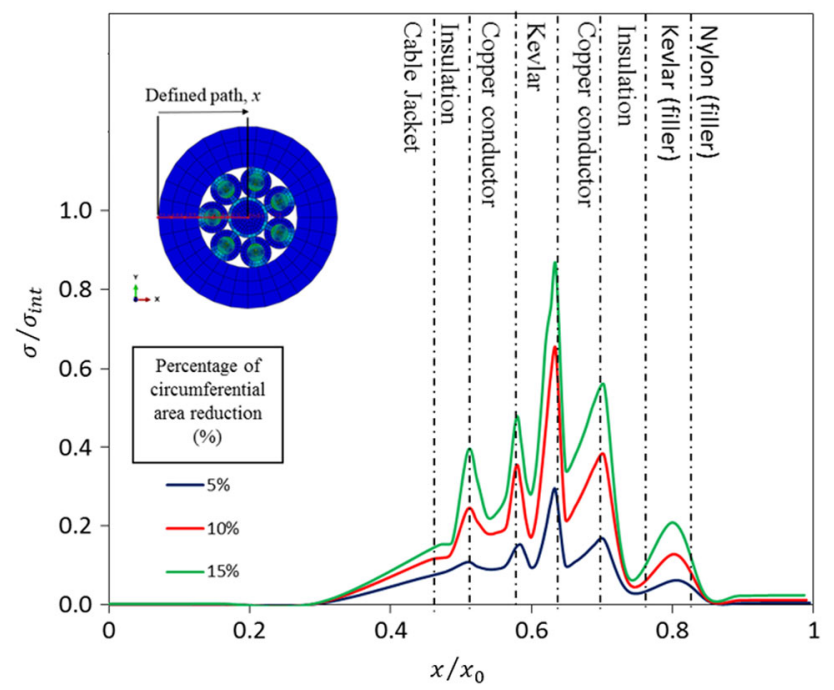

Fig. 12 The stress variation across a single-core wire affected by circumferential compression

The abrasive Kevlar strands could have formed a local pileup of broken remnants in the single-core wire that can induce a localized stress concentration on the copper conductors as the other components were still intact. The strength of copper conductors would be further weakened due to the compressive residual stress imparted by the cable jacket. To simulate the combined events, an assembly of RSM cable was developed with a locally reduced crosssectional area of $0,5,10$, and $15 \%$ at the center of the cable jacket. The state of stress due to local necking of the RSM cable jacket was examined, as shown in Fig. 12. It clearly shows a typical pattern of stress increase as the circumferential compression increases. However, the variation of the stress experienced by the components was shown to be non-uniform in the single-core wire due to the interaction with the other components.

The maximum stress experienced by the copper conductor is found to develop nearer to the central KevlarNylon composite filler, while the copper conductor nearer to the cable jacket shows a much lower stress level. For circumferential compressions of 5,10 , and $30 \%$, the peakto-peak stress difference for the single copper conductor closer to the Kevlar-Nylon core and the cable jacket is 10 , 30 , and $50 \%$, respectively. This indicates that the central Kevlar-Nylon composite filler is relatively more rigid than the cable jacket and causes the copper conductor nearer to it to experience much higher stress compared to the copper conductor nearer to the cable jacket. This can make the copper conductor nearer to the central Kevlar-Nylon filler more likely to develop the copper conductor critical intermittent failure stress.

The single-core wire damage evolution started with the breakage of the Kevlar strands within the single-core wire, followed by the breakage of the copper conductor which was responsible for the intermittent audio signal. At the same time, the cable jacket, nylon strands, and the insulation material absorbed large amounts of energy through a relatively large elongation as shown in Fig. 11. However, when the load was released back to a load below the intermittent failure load, it was observed that the broken copper conductor made contact again because the cable returned to its original state.

A comparison of the effect of tensile load and bending load on the assembled RSM cable is shown in Fig. 13 with the circles on the curves indicating the intermittent failure load. The intermittent failure load in finite element analysis was determined to be the load required to cause complete failure of copper conductors in RSM cable. The data presented in both figures were normalized with the experimental intermittent failure load. It is worth mentioning that the value of experimental intermittent failure load in tensile analysis, $P_{\text {int(tensile), }}$, is different from the one for bending, $P_{\text {int(bend) }}$. The abscissas were normalized as displacement, $U_{\mathrm{int}}$, and $D_{\mathrm{int}}$ for the tensile and bending loading conditions, respectively.

Figure 13a and $\mathrm{b}$ shows the finite element analysis for the tension and bending loads, respectively, compared to the experimental data. The intermittent failure load is indicated by the inset circle. The load in Fig. 13a and b was generalized by the appropriate intermittent failure load, $P_{\text {int }}$, but the tension intermittent load was about a third higher than the bending intermittent failure load.

The RSM cable under tension load in Fig. 13a showed that the intermittent failure load occurs at a longer extension in finite element analysis compared to the experimental data, unlike in Fig. 13b where the finite 

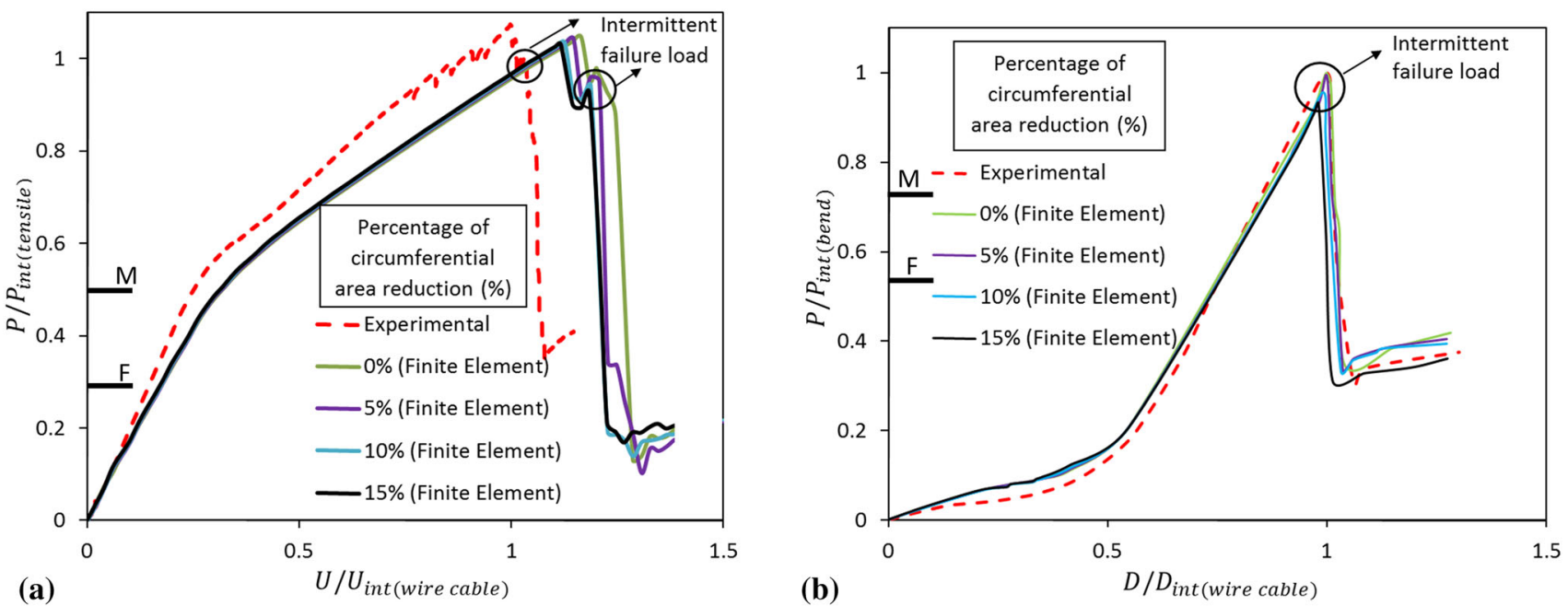

Fig. 13 Intermittent load of RSM cable affected by radial compression for (a) tension load and (b) bending load (horizontal line indicates the maximum human hand-grip strength for male (M) and female (F) as documented in [13])
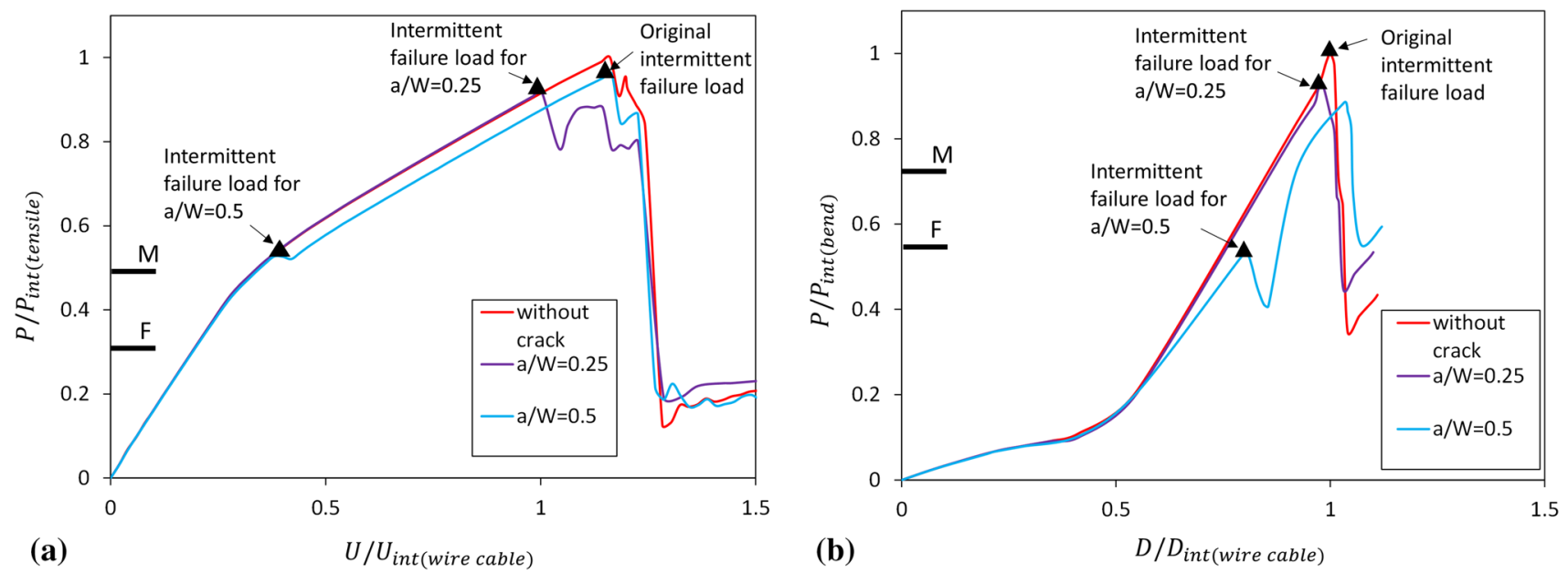

Fig. 14 Effect of crack depth on (a) tension load and (b) bending load for RSM cable (horizontal line indicates the maximum human hand-grip strength for male (M) and female (F) as documented in [13])

element result matched well with the experimental data. However, the intermittent loads from the finite element analysis were well matched to the experimental results for both the tension and bending loads. It was observed from the tension experimental analysis that the multi-core wires and the cable jacket experience an initial body translation between each other until a critical extension which caused an offset in the load-displacement curve and hence the reduced intermittent failure extension load. Although the extension in tensile analysis was different, the intermittent failure load agreed well with the experimental result. In the bending analysis, the body translation of multi-core wires to the cable jacket was not observed because the load applied was focused on a local area on the RSM wire cable unlike the tension analysis.
The maximum human hand-grip strength for male and female (indicated by the horizontal line) was normalized with the experimental intermittent failure load in each analysis, respectively. Figure 13 shows that the intermittent failure load was higher than the maximum human handgrip strength, and therefore the effect of local compression on the multi-core cable was unable to simulate a state of residual stress that could cause intermittent failure load due to normal handling load.

The effect of the micro surface cracks on the copper conductors for the tension and bending load configurations is shown in Fig. 14, where the intermittent failure load for the selected crack geometry is identified by a triangular marker. For both the tension (Fig. 14a) and bending loads (Fig. 14b), micro surface crack size has a profound effect on the strength 
of the multi-core wires. The increase in the micro surface crack depth causes a noticeable decrease of the intermittent failure load. The effect of the micro surface crack on bending load was more severe in tensile loading. However, deep cracks may result in intermittent failure in both tensile and bending loadings even under normal handling loads.

Unlike the effect of circumferential compression from the cable jacket, the presence of micro surface crack results in a more severe reduction of intermittent failure load. The estimated intermittent failure load for a crack of geometry $a / W=0.5$ for the tension was nearer to the maximum load applicable by an adult but much lower in the case of bending load that can be exerted by an adult using their hands (approximately $300 \mathrm{~N}$ ) as reported in [13].

\section{Conclusions}

The intermittent failure analysis of the RSM cable has shown that the failure was due to micro surface cracks that were found on the copper conductors. It is likely that this defect has been induced by the manufacturing processes of the copper conductor in the multi-core cable. However, there were a number of issues pertaining to the design of the RSM cable that should be considered to improve the integrity of the strength of the cable:

(1) The Kevlar-Nylon filler material must not induce a high stress on the copper conductors. A less rigid configuration should be adopted to offer a balanced stress level for the copper conductors.

(2) The shrink-fit of the cable jacket onto all the singlecore wires is a standard requirement in the design of the RSM cable. However, the shrink-fit will cause an inherent residual stress in the RSM cable. Therefore, the combination of micro cracks and residual stress may cause a premature degradation of the strength of the copper conductors.

(3) The intermittent failure load due to micro cracks was found to be more severe in bending load when compared to the tension loading. Therefore, the bending loading configuration must be used as a guide for the maximum limit of load applicable to the RSM cable.

(4) The typical design specification of an RSM cable must not only include the fatigue cycle specification but also include the quasi-static deformation load in tension and bending and combination of loadings to ensure zero tolerance to intermittent failure of the RSM cable.

Acknowledgments The authors acknowledge the funding through a grant P17C1-12 from CREST Malaysia and the funding from
Motorola Solutions Malaysia in the later stages of the project to Mr. Leong Karh Heng and Mr. Rizman Hariz Abdul Latiff. Thanks are also due to Mr. Kamaruddin Khalid and Mr. Alex Yeo Siang Chew who conducted the initial investigation of the RSM cable failure as part of their undergraduate project and Motorola Solutions Pte. Ltd. Malaysia for the supply of the RSM cable materials used in the testing. Finally, the ABAQUS finite element code was made available under an academic license from Dassault Systemes K.K., Japan.

\section{References}

1. A.K. Thiel, H. Stambaugh, Improving Firefighter Communications: Special Report: Federal Emergency Management Agency, US Fire Administration (1999)

2. U. DoD, Mil-STD-810F: Department of defense test method standard for environmental engineering considerations and laboratory tests. US Department of Defense $(D o D)(01.01$. 2000) (2000)

3. E.J. O'Neill, J. Halverson, Study of intermittent field hardware failure data in digital electronics. Sperry Univac Defense Systems, St. Paul, MN. NASA CR-159268 (1980)

4. S.-I. Nishida, Failure Analysis in Engineering Applications (Butterworth-Heinemann, Oxford, 1992)

5. H. Qi, S. Ganesan, M. Pecht, No-Fault-Found and Intermittent Failures in Electronic Products. Microelectron. Reliab. 48, 663674 (2008)

6. D.C. Green, Radio Communication (Longman, Harlow, 2000)

7. D. Onslow, Two-Way Radio Success, $3^{\text {rd }}$ edn. www. IntercomsOnline.com

8. Manual and Guidelines for Remote-Speaker-Microphone Users, Motorola Solutions Sdn. Bhd, Penang, Malaysia (2015)

9. United States Army Material Command, Engineering Design Handbook Electrical Wire and Cable (Books for Business, Canada, 2002)

10. UK Ministry of Defence, Wires Cords and Cables, ElectricalMetric Units Part 0: General Requirements and Test Methods for Qualification Approval, ed. (1992)

11. Internal Report: RSM Intermittent Failure, Motorola Solutions Sdn. Bhd., Penang, Malaysia (2012)

12. Specification for Wire Cables-3075335B08, Motorola Solutions Sdn. Bhd., Penang, Malaysia (2010)

13. H. Dreyfus and A. Tilley, "The Measure of Man and Woman: Human Factors in Design," ed: John Wiley \& Sons Inc, 1993

14. Initial SEM Analysis for RSM Cable Failure, Motorola Solutions Sdn. Bhd., Penang, Malaysia (2011)

15. P.J. Withers, M. Turski, L. Edwards, P.J. Bouchard, D.J. Buttle, Recent advances in residual stress measurement. Int. J. Press. Vessels Pip. 85, 118-127 (2008)

16. M.B. Prime, Cross-sectional mapping of residual stresses by measuring the surface contour after a cut. J. Eng. Mater. Technol. 123, 162-168 (2001)

17. A. Standard, "E8M-04," Standard Test Methods for Tension Testing of Metallic Materials (ASTM International, West Conshohocken, 2004)

18. ABAQUS, "V6.12," Tokyo, Japan. Analysis User's Guide, Dassault Systems (2013)

19. W.C. Orthwein, Clutches and Brakes: Design and Selection (CRC Press, Boca Raton, 2004)

20. Dupont. Hytrel Thermoplastic Polyester Elastomer Design Information

21. W.S. Temple, Crosslinked Polyethylene Jackets for Medium Voltage Cables. In: Fall 2010 IEEE PES ICC Meeting Subcommittee C-Cable Systems (2010)

22. Dupont. Properties and Processing of Dupont Kevlar Brand Yarn for Mechanical Rubber Goods 